\title{
Article \\ Simultaneous Determination and Health Risk Assessment of Four High Detection Rate Pesticide Residues in Pu'er Tea from Yunnan, China
}

\author{
Tao Lin ${ }^{1,2} \mathbb{D}$, Xing-Lian Chen ${ }^{1,2}$, Jin Guo ${ }^{3}$, Meng-Xia Li ${ }^{3}$, Yu-Feng Tang ${ }^{4}$, Mao-Xuan Li ${ }^{1,2}$, Yan-Gang Li ${ }^{1,2}$, \\ Long Cheng ${ }^{5}$ and Hong-Cheng Liu ${ }^{1,2, *}$
}

check for

updates

Citation: Lin, T.; Chen, X.-L.; Guo, J.; Li, M.-X.; Tang, Y.-F.; Li, M.-X.; Li, Y.-G.; Cheng, L.; Liu, H.-C. Simultaneous Determination and Health Risk Assessment of Four High Detection Rate Pesticide Residues in Pu'er Tea from Yunnan, China. Molecules 2022, 27, 1053. https:// doi.org/10.3390/molecules27031053

Academic Editor: James Barker

Received: 8 January 2022

Accepted: 1 February 2022

Published: 4 February 2022

Publisher's Note: MDPI stays neutral with regard to jurisdictional claims in published maps and institutional affiliations.

Copyright: (C) 2022 by the authors. Licensee MDPI, Basel, Switzerland. This article is an open access article distributed under the terms and conditions of the Creative Commons Attribution (CC BY) license (https:// creativecommons.org/licenses/by/ $4.0 /)$.
1 Quality Standards and Testing Technology Research Institute, Yunnan Academy of Agricultural Science, Kunming 650223, China; lintaonj@126.com (T.L.); chen544141152@163.com (X.-L.C.); limx1970@126.com (M.-X.L.); yglikm@sina.com (Y.-G.L.)

2 Laboratory of Quality and Safety Risk Assessment for Agro-Products (Kunming), Ministry of Agriculture and Rural Affairs, Kunming 650223, China

3 School of Medicine, Yunnan University of Business Management, Kunming 650106, China; gjin0111@163.com (J.G.); limengxia2022@163.com (M.-X.L.)

4 College of Agronomy and Life Sciences, Zhaotong University, Zhaotong 657000, China; tyfsunny@163.com

5 SCIEX Analytical Instrument Trading Co., Ltd., Shanghai 200335, China; long.cheng@sciex.com

* Correspondence: liuorg@163.com; Tel./Fax: +86-871-6514-9900

\begin{abstract}
Four pesticides with a high detection rate in Pu'er tea have been determined by a QuEChERS (quick, easy, cheap, effective, rugged, safe) method with multiwalled carbon nanotubes (MWC$\mathrm{NTs}$ ), and combined ultrahigh-performance liquid chromatography-triple quadrupole linear ion trap-tandem mass spectrometry (UHPLC-QTRAP-MS/MS). MWCNs have been compared with other common purification materials, and found to be superior. The matrix effect was systematically studied, and the results show that the MWCNs can quickly and effectively reduce matrix interference values, which were in the range from -17.8 to 13.8 . The coefficients $\left(R^{2}\right)$ were greater than 0.99 , with the limit of quantification ranging from 0.1 to $0.5 \mu \mathrm{g} / \mathrm{kg}$, and the recovery rate ranging from $74.8 \%$ to $105.0 \%$, while the relative standard deviation (RSD) ranged from $3.9 \%$ to $6.6 \%$. A total of 300 samples, taken from three areas in which Yunnan Pu'er tea was most commonly produced, tested for four pesticides. The results show that the detection rate of tolfenpyrad in Pu'er tea was $35.7 \%$, which is higher than other pesticides, and the lowest was indoxacarb, with 5.2\%. The residual concentrations of chlorpyrifos, triazophos, tolfenpyrad and indoxacarb ranged from 1.10 to $5.28,0.014$ to 0.103 , 1.02 to 51.8 , and 1.07 to $4.89 \mathrm{mg} / \mathrm{kg}$, respectively. By comparing with China's pesticide residue limits in tea (GB 2763-2021), the over standard rates of chlorpyrifos, tolfenpyrad, and indoxacarb were $4.35 \%, 0.87 \%$ and $0 \%$, respectively. The risk assessment result obtained with the hazard quotient (HQ) method shows that the HQ of the four pesticides was far less than one, indicating that the risk is considered acceptable for the four pesticides in Pu'er tea. The largest HQ was found for tolfenpyrad, 0.0135 , and the smallest was found for indoxacarb, 0.000757 , but more attention should be paid to tolfenpyrad in daily diets in the future, because its detection rate, and residual and residual median were all relatively high.
\end{abstract}

Keywords: high detection rate pesticides; multiwalled carbon nanotubes; risk assessment; Pu'er tea

\section{Introduction}

$\mathrm{Pu}^{\prime}$ er tea is one of the most famous teas in China because of its various health benefits, such as lowering blood fat [1], weight loss [2], and antibacterial properties [3], as well as its unique taste and aroma [4,5]. It is beloved by consumers all over the world. With the increasing consumption of $\mathrm{Pu}^{\prime}$ er tea [6], wild-grown tea raw materials can no longer meet the current sharp increase in consumption. To meet these demands, large-scale industrial farming of these ingredients, as well as the concurrent use of chemical pesticides, has been 
implemented. However, the indispensable and excessive use of pesticides has resulted in $\mathrm{Pu}^{\prime}$ er tea containing many pesticide residues, which have potential risks to the health of its consumers [7].

Tolfenpyrad and indoxacarb are commonly used pesticides for Pu'er tea production or during tea plantation. They are effective in controlling Empoasca pirisuga, Matumura, and mite pests [8,9]. Indoxacarb is a low-toxicity pesticide [10], while tolfenpyrad is more toxic to aquatic organisms [11]. Chlorpyrifos and triazophos are also high detection rate pesticides. They are currently banned in China because they were detected in quantities exceeding the standard in vegetables, and also because of their moderate toxicity [12]. In addition, lower values of the acceptable daily intake (ADI) of chlorpyrifos and triazophos have been stipulated in the National Food Safety Standard-Maximum Residue Limits for Pesticides in Food (GB 2763-2021) [13].

In GB 2763-2021, the detection method for tolfenpyrad and indoxacarb in tea is liquid chromatography-tandem mass spectrometry, which requires the use of solid phase extraction cartridges for purification, and the use of toxic toluene as the elution solvent. This method is more harmful to the human body and the environment. The methods for the determination of chlorpyrifos and triazophos mainly include liquid chromatographytandem mass spectrometry [14-16] and gas chromatography-mass spectrometry [17-19], but the detection period of gas chromatography-mass spectrometry is relatively long, which means that it cannot meet the current requirements for rapid high-sensitivity determination. When liquid chromatography-tandem mass spectrometry is used for the determination of low-level pesticides in complex matrices, false-positive detections may occur due to the interference of impurities [20,21]. The literature has reported the use of the QuEChERS method for the determination of pesticides in tea. Most of these methods, such as primary secondary amine (PSA), graphitized carbon black (GCB), and $\mathrm{C}_{18}$, are commonly used purification fillers [15,22-24]. However, there are few articles documenting the use of the QuEChERS method in Pu'er tea [25]. The same can be said for MWCNTs.

The complex matrix of Pu'er tea contains a variety of chemical components, which can affect the detection of pesticides. Conducting a qualitative analysis on the low levels of pesticides in Pu'er tea was difficult, especially when there was interference from the transitions or a shift in retention times [26,27]. Linear ion trap mass spectrometry combined a triple-quadruple (Qtrap) scanning functionality with sensitive ion trap scans. These additional Qtrap scan functions greatly enhanced the performance of screening, confirmation, and identification [20,21]. The detection rate of these four pesticide residues in Pu'er tea is high, indicating that human exposure to pesticides is becoming a more serious issue [28]. However, the current use of residue limits is not reasonable for the safety evaluation of $\mathrm{Pu}^{\prime}$ er tea, and the lack of a multi-residue assessment method is unfavorable for the risk assessment of Pu'er tea.

In this study, the presence of four pesticides with a high detection rate in $\mathrm{Pu}^{\prime} \mathrm{er}$ tea was established using the QuEChERS method, combined with UHPLC/QTRAP-MS/MS and MWCNTs purifying filler. The established measurement method was used to determine the presence of pesticides in 300 samples from the three areas in which Yunnan Pu'er tea is most commonly produced. A risk assessment was carried out using the hazard quotient method [29]. The results provide basic data for consumers regarding the safety of drinking $\mathrm{Pu}^{\prime}$ er tea.

\section{Materials and Methods}

\subsection{Sample Collection and Preparation}

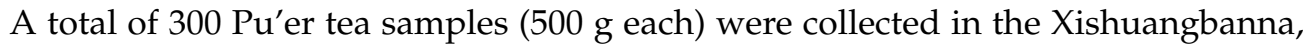
$\mathrm{Pu}^{\prime}$ er and Lincang regions of Yunnan Province. A total of 100 samples from each region were collected. Sample collection was conducted in May 2021. All Pu'er tea samples were collected from supermarkets or farmers' markets with the permission of local management personnel, and all the supermarkets or farmers' markets are legally registered with their local authority. Collected samples were immediately transported to the laboratory and 
stored in the original sealed packaging at $4{ }^{\circ} \mathrm{C}$ in the dark. Sampling was carried out according to the Chinese standard (GB/T 8302-2013), established by the Standardization Administration of China [30].

\subsection{Chemicals and Reagents}

Chlorpyrifos, indoxacarb, triazophos, and tolfenpyrad standards were used at a concentration of $1000 \mathrm{mg} / \mathrm{L}$, in which chlorpyrifos and triazophos were dissolved in acetone, and indoxacarb and tolfenpyrad were dissolved in methanol, purchased from Agro-Environmental Protection Institute, Ministry of Agriculture (Tianjin, China). Methanol and acetonitrile of HPLC grade were obtained from Merck KGaA (Darmstadt, Germany). Highly purified water was prepared by a Milli-Q water purification system (Bedford, MA, USA). Ammonium formate ( $\geq 99.995 \%$ ) was purchased from Millipore Sigma Company (St Louis, MO, USA). Analytical reagent grade, including anhydrous sodium chloride $(\mathrm{NaCl})$ and magnesium sulfate $\left(\mathrm{MgSO}_{4}\right)$, were obtained from Sinopharm Chemical Reagent (Beijing, China). MWCNTs (10-20 nm diameter, 10-30 $\mu \mathrm{m}$ length) were provided by Nanjing XFNANO Materials Tech Co., Ltd. (Nanjing, China). Purification materials, including primary secondary amine (PSA), Florisil, graphitized carbon black (GCB), $\mathrm{NH}_{2}$, and $\mathrm{C}_{18}$ with a diameter of $50 \mu \mathrm{m}$, were bought from Dikma Technologies Inc. (Beijing, China).

\subsection{Sample Preparation and Analysis}

\subsubsection{Sample Preparation Method}

The experiment was performed in accordance with the regulations (NY/T 789-2004) established by the Ministry of Agriculture and Rural Affairs of the People's Republic of China. The Pu'er tea samples (about $300 \mathrm{~g}$ ) were ground and passed through a $200 \mathrm{~mm}$ polyethylene sieve. Two grams of tea leaves was weighed and placed into a $50 \mathrm{~mL}$ centrifuge tube. Ten milliliters of water were added, vortexed for half a minute, and allowed to stand for $30 \mathrm{~min}$. After the water in the Pu'er tea was fully soaked, $15 \mathrm{~mL}$ of acetonitrile was added and vortexed for $2 \mathrm{~min}$. Then, $3 \mathrm{~g}$ sodium chloride was added, vortexed for half a minute and centrifuged at $5000 \mathrm{r} / \mathrm{min}$. Two milliliters of the upper extract were taken out and placed into another $10 \mathrm{~mL}$ centrifuge tube. A total of $300 \mathrm{mg}$ of anhydrous magnesium sulfate and $40 \mathrm{mg}$ of multi-walled carbon nanotubes were added, vortexed for half a minute and centrifuged at $5000 \mathrm{r} / \mathrm{min}$. The supernatant was filtered through a $0.22 \mu \mathrm{m}$ filter membrane before analysis.

\subsubsection{Instrumental Analysis Method}

Sample analyses were performed with an AB Sciex QTRAP 5500 mass spectrometer (MS/MS) (Framingham, MS, USA) equipped with 1290II Infinity UHPLC (Agilent Technology, Santa Clara, CA, USA) and Waters ACQUITY UPLC BEH T3 column $(2.1 \times 50 \mathrm{~mm}$, $1.8 \mu \mathrm{m}$, Waters, MA, USA). Solvents A (1 mM ammonium formate in ultrapure water with $0.1 \%$ formic acid) and $\mathrm{B}$ (methanol) were used at a flow rate of $0.3 \mathrm{~mL} / \mathrm{min}$ with the following gradient: $5 \% \mathrm{~B} \rightarrow 95 \% \mathrm{~B}(0 \sim 3.5 \mathrm{~min}) \rightarrow 95 \% \mathrm{~B}(3.5 \sim 5.5 \mathrm{~min}) \rightarrow 5 \% \mathrm{~B}$ $(5.5 \sim 5.7 \mathrm{~min}) \rightarrow 5 \%$ B (5.7 8.0 min). The injection volume was $1 \mu \mathrm{L}$.

The electrospray ionization (ESI) source was operated in positive $\left(\mathrm{ESI}^{+}\right)$mode for forming [analyte $+\mathrm{H}]^{+}$ions. Analyte ion transitions used for qualification and quantitation were monitored using multiple reaction monitoring (MRM) mode. Ion source conditions were as follows: ionspray voltage, $5500 \mathrm{~V}$; heating gas temperature, $550{ }^{\circ} \mathrm{C}$; curtain gas flow rate, $20 \mathrm{~L} / \mathrm{h}$; nebulizing gas flow rate, $55 \mathrm{~L} / \mathrm{h}$; heating gas flow rate, $55 \mathrm{~L} / \mathrm{h}$. The identification of proper ion transitions (precursor ion $>$ product ion) of each pesticide and the optimization of a number of MS/MS parameters, including declustering potential (DP) and collision energy (CE), were performed with a syringe pump, providing a constant flow of the standard solution $(0.1 \mu \mathrm{g} / \mathrm{mL})$ of four pesticides to the MS/MS at a flow rate of $10 \mu \mathrm{L} / \mathrm{min}$. An enhanced product ion (EPI) scan mode was applied in QTrap. EPI mode was acquired from 50 to $600 \mathrm{amu}$ with a scan speed of 10,000 amu/s. The CE and 
collision energy spread (CES) were $35 \mathrm{~V}$ and $15 \mathrm{~V}$, respectively. The other parameters for the detection of the four pesticides are shown in Table 1.

Table 1. UHPLC-MS/MS parameters for detection of the four pesticides.

\begin{tabular}{cccccc}
\hline Pesticide & Ionization Mode & Precursor Ion/(m/z) & Product Ion/(m/z) & DP/(Volts) & CE/(Volts) \\
\hline Chlorpyrifos & $\mathrm{ESI}^{+}$ & 350.0 & $198.0^{*} / 96.9^{\#}$ & 60 & $25 / 40$ \\
Triazophos & $\mathrm{ESI}^{+}$ & 314.0 & $162.0^{*} / 119.1^{\#}$ & 55 & $25 / 50$ \\
Tolfenpyrad & $\mathrm{ESI}^{+}$ & 384.0 & $197.1^{*} / 154.1^{\#}$ & 60 & $35 / 55$ \\
Indoxacarb & $\mathrm{ESI}^{+}$ & 528.0 & $249.1^{*} / 293.1^{\#}$ & 60 & $25 / 22$ \\
\hline
\end{tabular}

* quantitative ion, \# qualitative ion.

\subsubsection{Method Validation}

The quantification limits, linear ranges and correlation coefficients of each pesticide in Pu'er teas were determined according to SANTE/ 12682/2019 [31]. According to the actual sensitivity of the instrument for detecting the four pesticides, $0.01-5.0 \mathrm{ng} / \mathrm{mL}$ was selected as the linear range of the four pesticides, including $0.1,0.2,0.5,1.0,2.0$ and $5.0 \mathrm{ng} / \mathrm{mL}$. The limit of quantification (LOQ) and limit of detection (LOD), estimated at signal-tonoise ratios of 10 and 3, were evaluated by spiked blank Pu'er tea samples for quality control. Recovery and reproducibility experiments were conducted in 6 replicates, each at 3 concentration levels (LOQs, $5 \times$ LOQs, $10 \times$ LOQs). The intraday and intraday precision were determined by recovering 3 concentration levels (LOQs, $5 \times$ LOQs, $10 \times$ LOQs) at different time points on the same day, and on different days of the week, with 6 replicates for each added concentration. Their stability was assessed by calculating the RSD.

\subsection{Matrix Effect}

The slopes of calibration curves of 4 pesticides in different purification materials and organic solvents were studied to evaluate matrix effects. Matrix effects (ME) were measured according to Equation (1) [32], as follows:

$M E=($ slope of solvent standard/slope of matrix matched standard -1$) \times 100 \%$.

This means that the matrix is inhibited when $\mathrm{ME}<0$ and the matrix is enhanced when $\mathrm{ME}>0$. There is a weak matrix effect when the absolute value of ME is $0-20 \%$, a medium matrix effect when the absolute value of $\mathrm{ME}$ is $20-50 \%$, and a strong matrix effect when the absolute value of $\mathrm{ME}$ is $50 \%$.

\subsection{Risk Assessment}

The pesticide hazard quotient (HQ) of long-term potential health effects was evaluated as Equation (2). The HQ can be interpreted as follows: $\mathrm{HI}<1$, the risk is considered acceptable; $\mathrm{HI} \geq 1$, there is an unacceptable risk [29].

$$
\mathrm{HQ}=\mathrm{EDI} / \mathrm{ADI}
$$

where ADI ( $\mathrm{mg} \mathrm{kg}^{-1} \mathrm{bw}$ ) is the acceptable daily pesticide intake (the ADI of every pesticide can be found in GB 2763-2021) and EDI is the estimated daily intake, which was calculated as Equation (3) as follows:

$$
\mathrm{EDI}=\mathrm{C} \times \mathrm{D} \times \mathrm{T} / \mathrm{Bw}
$$

where $\mathrm{C}$ is the average residual content of a pesticide in $\mathrm{Pu}$ 'er tea $(\mathrm{mg} / \mathrm{kg}), \mathrm{D}$ is the amount of tea consumed per day $(\mathrm{g}), \mathrm{T}$ is the transfer rate of pesticide residue from made tea to tea infusion, and Bw is the body weight $(\mathrm{kg})$. 


\section{Results}

\subsection{Optimization of Instrument Conditions}

In this experiment, a T3 chromatographic column, with better separation performance than $C_{18}$, was selected [33,34], which can complete the analysis of a sample in $8 \mathrm{~min}$. At the same time, the $\mathrm{ESI}^{+}$mode was selected for the determination of four pesticides; the peak shapes and the response of the four pesticides were improved by adding a certain amount of formic acid and ammonium acetate in the mobile phase. The chromatograms of the four pesticides are shown in Figure 1.

\subsection{QTrap System}

EPI (enhanced product ion) mode is a common mode in QTrap scanning. In this mode, the parent ion of the target compound is first selected in the first quadrupole (Q1), and all other ions are filtered out. The parent ions are generated via collisionally activated dissociation (CAD) in the collision cell of the second quadrupole (Q2), and the fragment ions are captured and enriched by the ion trap to obtain an enhanced secondary ion full scan mass spectrum. Under low-concentration conditions, QTrap can be used to obtain high-quality secondary mass spectra. At the same time, the target compound can be confirmed by matching the secondary mass spectra of the standard and positive samples. Figure 2 shows the secondary mass spectra obtained by using QTrap for four pesticides. As shown in Figure A1 (Appendix A), the secondary mass spectra of the positive sample and the standard were compared, and the comparison results show that the matching degree of the four pesticides was greater than $95 \%$, indicating that the use of QTrap can further improve the sensitivity, confirmation and reliability of pesticide detection for Pu'er tea.

\subsection{Matrix Effects}

The slopes of the standard curves prepared by acetonitrile extract, acetonitrile extract after purification with different purification materials (MWCNTs, $\mathrm{NH}_{2}, \mathrm{PSA}$, florisil, $\mathrm{C}_{18}$, and GCB), and methanol have been compared. According to the calculation formula of the matrix effect, the standard curve made of methanol should be used as the benchmark; the closer the slope of other curves is to methanol, the smaller the matrix effect will be. The four pesticides are shown in Figure 3. For chlorpyrifos, the slopes of the GCB and MWCNT purification solutions were closer to methanol. For triazophos, the slopes of the MWCNT and $C_{18}$ purification solutions were closer to methanol. For indoxacarb, the slopes of the MWCNT and PSA purification solutions were relatively close to those of methanol. For tolfenpyrad, the slopes of the MWCNT and $\mathrm{C}_{18}$ purification solutions were relatively close to those of methanol. Although florisil, PSA, and $\mathrm{C}_{18}$ were effective in reducing the matrix effect of triazophos, indoxacarb, and tolfenpyrad, interfering substances, such as pigments, polyphenols, and acidic components in Pu'er tea, can be effectively removed by MWCNTs, which can effectively reduce the matrix effect of the four pesticides $[14,35,36]$. Therefore, MWCNT was selected as the suitable purification material for the four pesticides.

\subsection{Optimization of the Amount of $\mathrm{MWCNTS}$ and $\mathrm{MgSO}_{4}$}

The effects of MWCNT addition $(10,20,30,40,50$, and $60 \mathrm{mg})$ on the recovery of four pesticides were compared. Figure 4 shows that when the amount of $\mathrm{MgSO}_{4}$ added was $300 \mathrm{mg}$, with the increase in the addition of MWCNTs, the recovery rates of chlorpyrifos, tolfenpyrad and triazophos exhibited a decreasing trend. However, when the addition amount was $40 \mathrm{mg}$, the recovery rates of chlorpyrifos and tolfenpyrad were close to stable, while the recovery rate of indoxacarb was largely unaffected by the addition amount of MWCNTs. Therefore, an addition of $40 \mathrm{mg}$ MWCNTs was considered to be the best choice. 

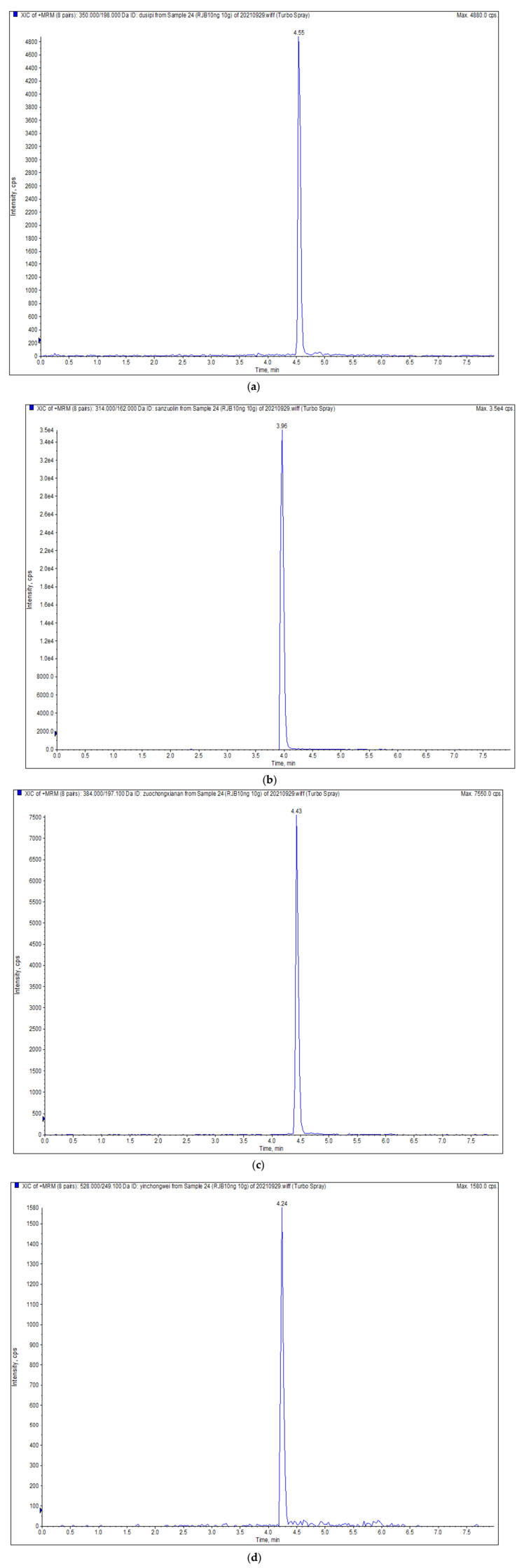

Figure 1. Chromatograms of 4 pesticides. (a) Chlorpyrifos; (b) triazophos; (c) tolfenpyrad; (d) indoxacarb. 

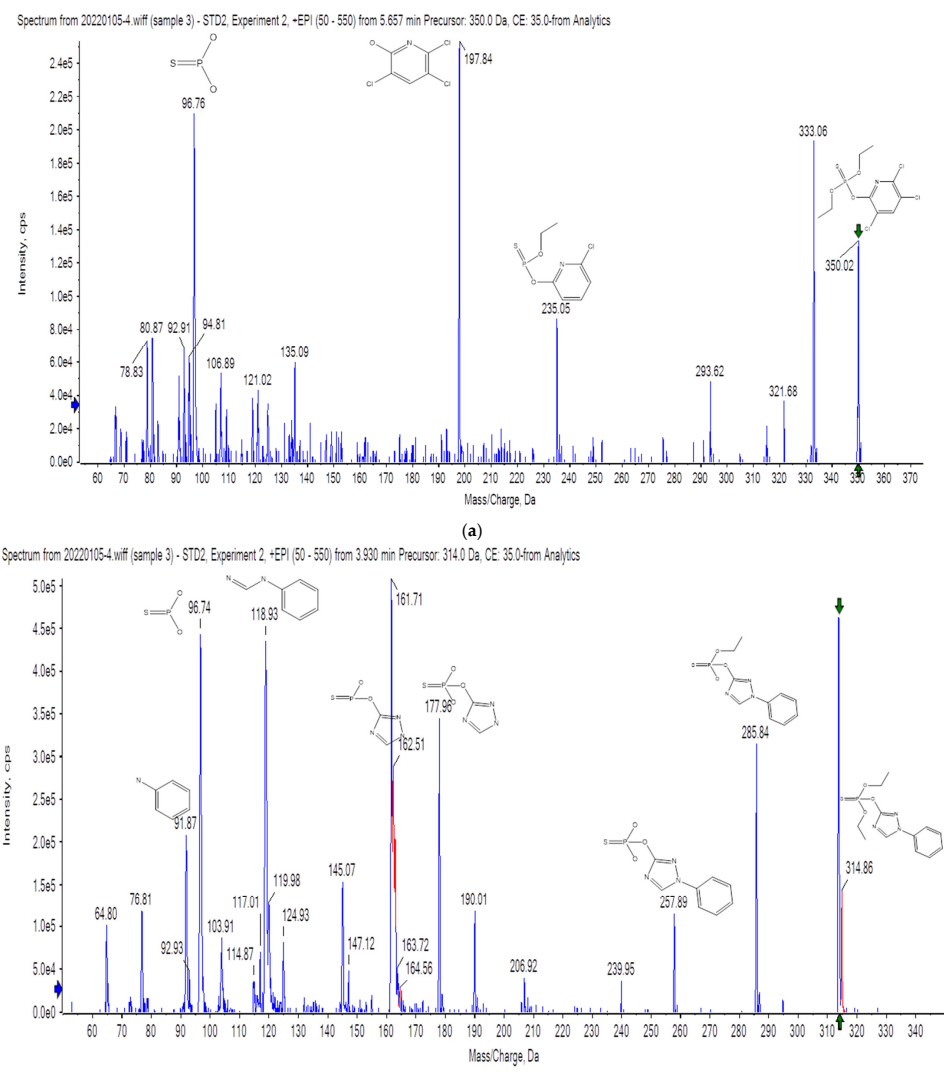

(b)

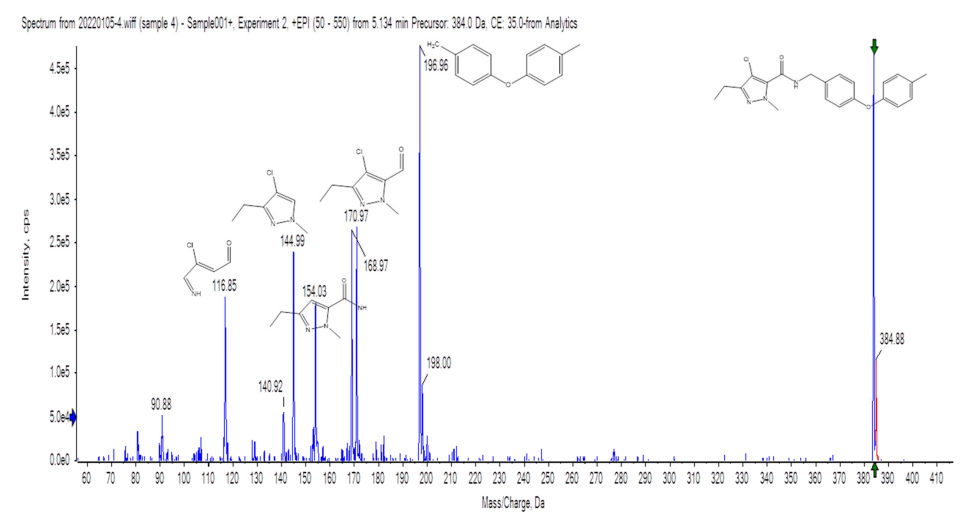

(c)

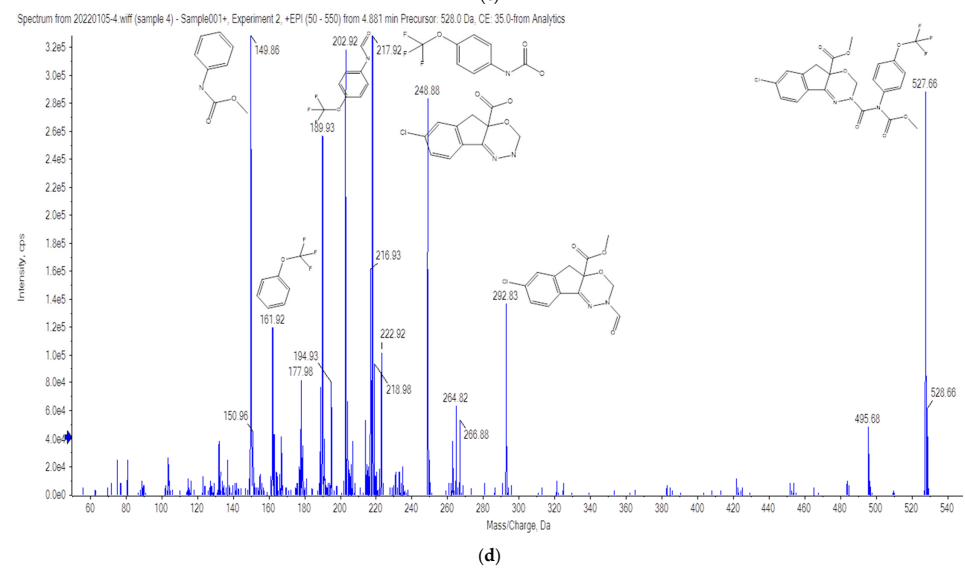

Figure 2. QTrap spectrum (EPI) of positive samples and standard for four pesticides in Pu'er tea. (a) Chlorpyrifos; (b) triazophos; (c) tolfenpyrad; (d) indoxacarb. 


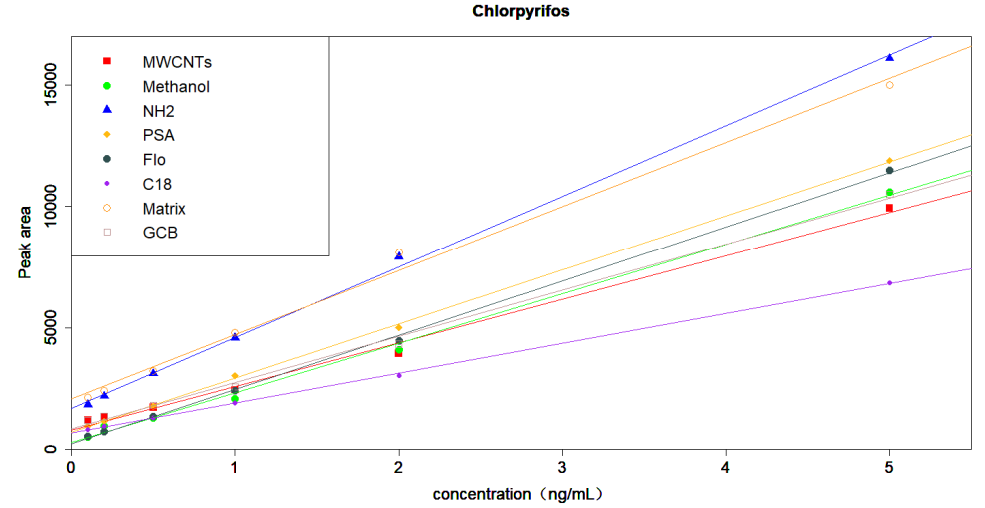

(a)

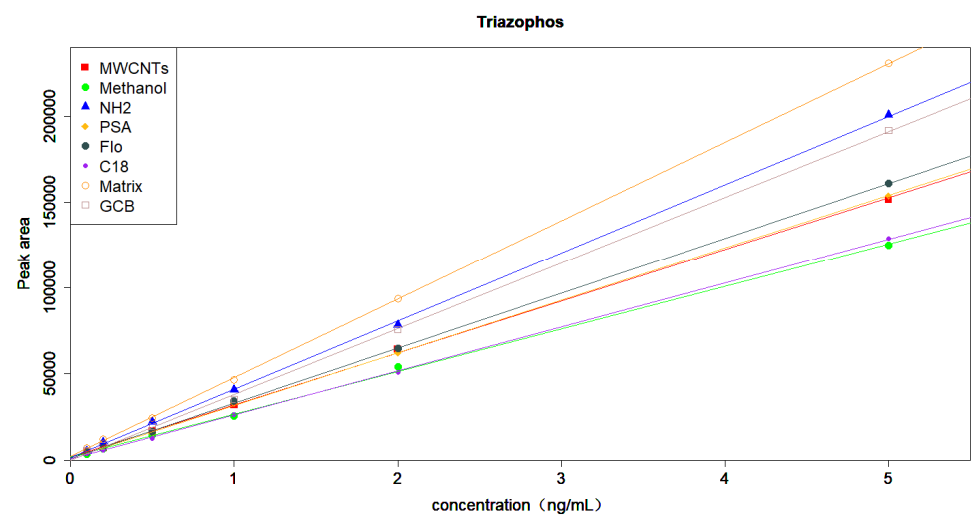

(b)

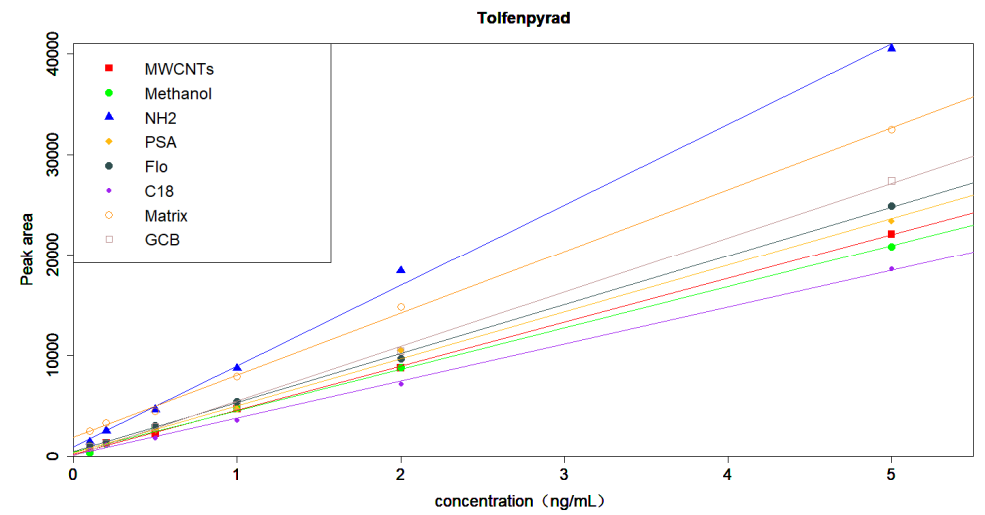

(c)

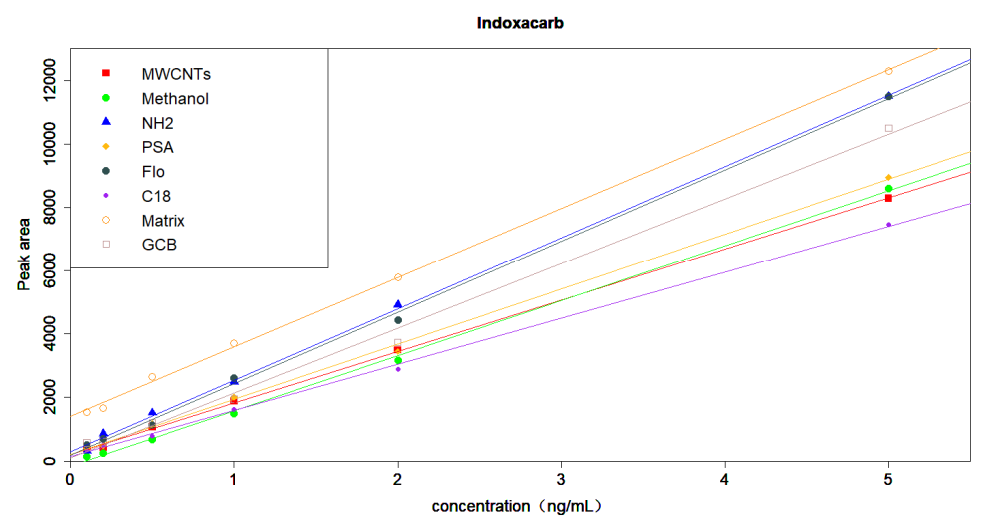

(d)

Figure 3. Standard curve of matrix preparation with different preparation methods. (a) Chlorpyrifos; (b) triazophos; (c) tolfenpyrad; (d) indoxacarb. 


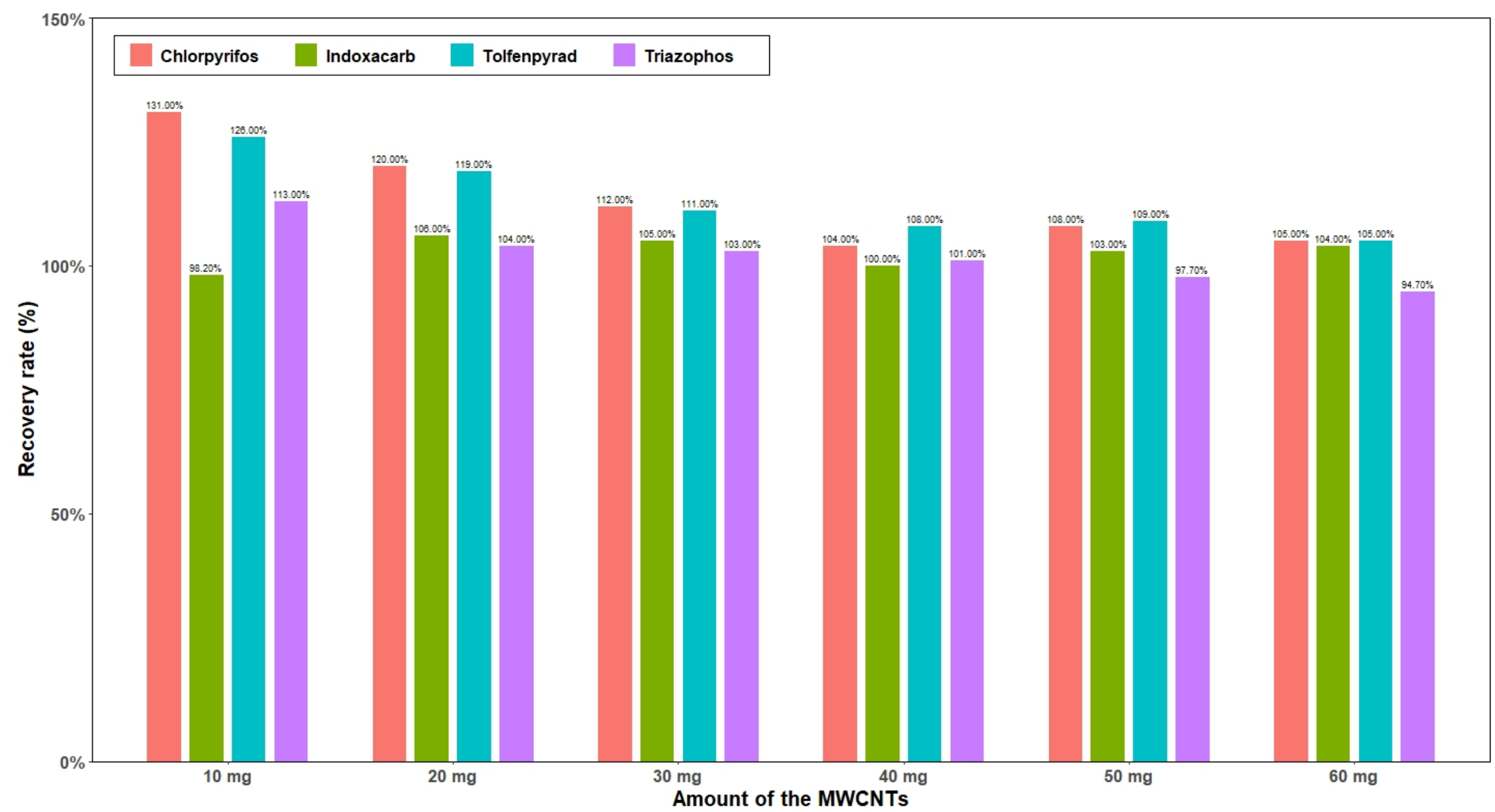

Figure 4. The influence of different contents of MWCNTs on the recovery rate of four pesticides. 
On the other hand, the effects of different amounts of $\mathrm{MgSO}_{4}(50,100,200,300,400$, $500 \mathrm{mg}$ ) on the recovery effects of the four pesticides were also compared. In Figure 5, when the added amount of MWCNTs was $40 \mathrm{mg}$, as the amount of $\mathrm{MgSO}_{4}$ added increases, the recovery rate of chlorpyrifos and tolfenpyrad continues to increase. It is possible that the added amount of $\mathrm{MgSO}_{4}$ better absorbs water in the solution environment, improving the extraction rate. Triazophos has little effect on the added amount. The recovery of indoxacarb increases with the increase in the added amount. In general, the addition of too little or too much $\mathrm{MgSO}_{4}$ will affect the recovery of the target compound. When the added amount was $200-300 \mathrm{mg}$, a better recovery effect was obtained. Taking this into consideration, we decided that $300 \mathrm{mg}$ was the best option.

\subsection{Method Validation}

As shown in Table 2, linearity was studied in the range of $0.01-5.0 \mu \mathrm{g} / \mathrm{L}$ for four pesticides by matrix-matched standard calibration with a good linear range. The described method was tested for limits of quantification of the four pesticides. Table 2 shows that the limits of quantitation (LOQs), the limits of detection (LODs), and the matrix effects of the pesticides' values were in the range of $0.10-0.50 \mu \mathrm{g} / \mathrm{kg}$ and $-17.8-13.8$, respectively. The LOQs in this study were compared with the LOQs in other studies for these four pesticides in tea, and the results showed that this method has greater detection sensitivity than the methods used in other studies [15,37-39].

Table 2. Linear ranges, correlation coefficients $\left(R^{2}\right)$, limits of quantitation (LOQs), limit of detection (LODs) and matrix effect of four pesticides.

\begin{tabular}{cccccc}
\hline Pesticide & Linear Ranges $(\mu \mathrm{g} / \mathrm{L})$ & $\boldsymbol{R}^{\mathbf{2}}$ & LOQ $(\mu \mathrm{g} / \mathbf{k g})$ & $\mathbf{L O D}(\boldsymbol{\mu g} / \mathbf{k g})$ & $\mathbf{M E} \%$ \\
\hline Chlorpyrifos & $0.01-5.0$ & 0.9944 & 0.10 & 0.03 & 13.8 \\
Triazophos & $0.01-5.0$ & 0.9997 & 0.50 & 0.15 & -17.8 \\
Tolfenpyrad & $0.01-5.0$ & 0.9996 & 0.20 & 0.06 & -5.8 \\
Indoxacarb & $0.01-5.0$ & 0.9995 & 0.50 & 0.15 & 7.3 \\
\hline
\end{tabular}

The recoveries of these four pesticides in Pu'er tea were determined by carrying out six consecutive extractions $(n=6)$ at three concentration levels (LOQs, $5 \times$ LOQs, $10 \times$ LOQs). The values were calculated using blank Pu'er tea matrix-matched calibration standards, as shown in Table 3, which details the recovery and relative standard deviation data for the four pesticides. The recoveries of the four pesticides in Pu'er tea were in the range of $74.8-105.0 \%$, with relative standard deviations (RSDs) in the range of $3.9-6.6 \%$.

\subsection{Pesticide Residues in Pu'er Tea}

According to Table 4, the detection rate of tolfenpyrad among the four pesticides was relatively high $(35.7 \%)$, and the lowest was indoxacarb (5.2\%). By comparing these data with China's pesticide residue limits in tea (GB 2763-2021), the over standard rates of 25 chlorpyrifos, tolfenpyrads, and indoxacarbs were $4.35 \%, 0.87 \%$, and $0 \%$, respectively. Because different countries have different pesticide residue limits (Table 5) [40], four pesticides in $\mathrm{Pu}^{\prime}$ er tea that do not meet the national standards of other nations will not be discussed in this study. However, by comparing these limit standards with those of other countries and regions, especially Japan, England, and the European Union, which import large amounts of $\mathrm{Pu}^{\prime}$ er tea from China, it is clear that special attention should be paid to their limit standards, as they are stricter than those in China.

\subsection{Consumer Exposure Assessment}

According to Table 6, the HQ of the four pesticides was far less than one, which indicates that the risk of these four pesticides in $\mathrm{Pu}^{\prime} \mathrm{er}$ tea was considered acceptable. The HQ of these pesticides ranges from 0.000757 to 0.0135 , of which the highest HQ was tolfenpyrad, with the highest detection rate, while the smallest was indoxacarb, with the lowest detection rate. 


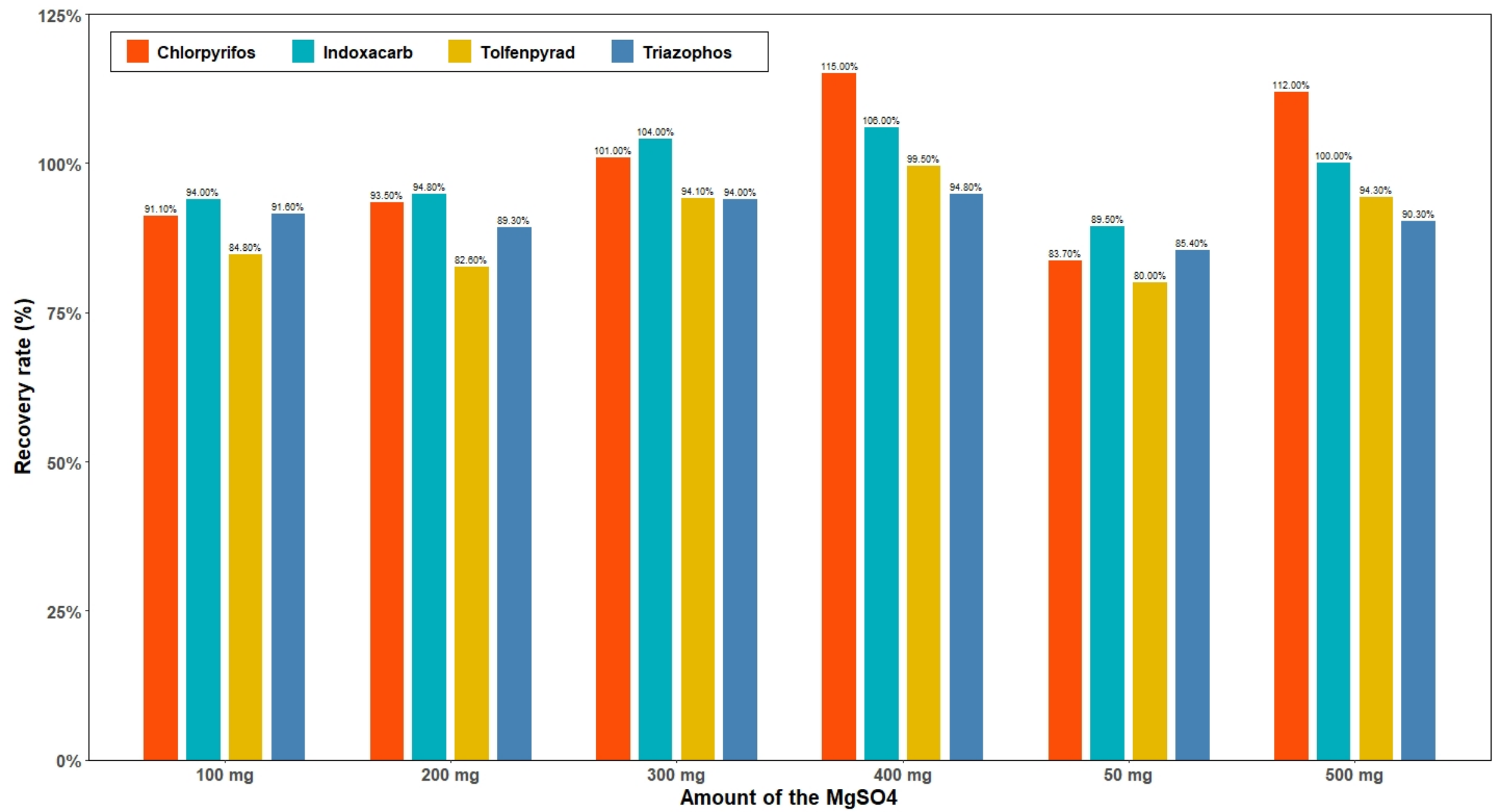

Figure 5. The influence of different contents of $\mathrm{MgSO}_{4}$ on the recovery rate of four pesticides. 
Table 3. Recoveries, relative standard deviations, intraday and interday precisions of four pesticides.

\begin{tabular}{ccccc}
\hline Pesticide & $\begin{array}{c}\text { Spiked Amount } \\
(\boldsymbol{\mu g} / \mathbf{k g})\end{array}$ & $\begin{array}{c}\text { Recovery } \\
(\mathbf{\%}) / \text { RSD }(\%)\end{array}$ & $\begin{array}{c}\text { Intraday } \\
\text { Precision (\%) }\end{array}$ & $\begin{array}{c}\text { Interday } \\
\text { Precision (\%) }\end{array}$ \\
\hline \multirow{3}{*}{ Chlorpyrifos } & 0.10 & $85.4 / 5.9$ & 4.5 & 5.2 \\
& 0.50 & $103.0 / 6.4$ & 4.2 & 5.8 \\
Triazophos & 1.00 & $105.0 / 5.3$ & 3.7 & 4.7 \\
& 0.50 & $74.8 / 5.3$ & 3.7 & 4.1 \\
Tolfenpyrad & 2.50 & $92.9 / 4.7$ & 4.3 & 4.5 \\
& 5.00 & $96.4 / 3.9$ & 3.9 & 5.5 \\
& 0.20 & $81.3 / 6.5$ & 4.9 & 3.5 \\
Indoxacarb & 1.00 & $97.3 / 6.6$ & 4.7 & 4.5 \\
& 2.00 & $101.0 / 5.9$ & 3.9 & 3.6 \\
& 0.50 & $90.4 / 6.7$ & 4.7 & 4.4 \\
\hline
\end{tabular}

Table 4. Pesticide residues detected in Pu'er tea.

\begin{tabular}{ccccc}
\hline Pesticide & Detection Rate $\mathbf{\%} \%$ & $\begin{array}{c}\text { Range of Detected Content } \\
(\mathbf{m g} / \mathbf{k g})\end{array}$ & $\begin{array}{c}\text { Mean Residue Level } \\
(\mathbf{m g} / \mathbf{k g})\end{array}$ & $\begin{array}{c}\text { Median Residue Level } \\
(\mathbf{m g} / \mathbf{k g})\end{array}$ \\
\hline Chlorpyrifos & 12.2 & $1.10-5.28$ & 2.14 & 1.62 \\
Triazophos & 10.4 & $0.014-0.103$ & 0.049 & 0.046 \\
Tolfenpyrad & 35.7 & $1.02-51.8$ & 11.6 & 5.01 \\
Indoxacarb & 5.2 & $1.07-4.89$ & 2.84 & 2.96 \\
\hline
\end{tabular}

Table 5. The maximum residue limits for four pesticides in tea in different countries and regions.

\begin{tabular}{|c|c|c|c|c|c|c|c|c|}
\hline Pesticide & $\begin{array}{l}\text { China } \\
(\mathrm{mg} / \mathrm{kg})\end{array}$ & $\begin{array}{l}\text { England } \\
(\mathrm{mg} / \mathrm{kg})\end{array}$ & $\begin{array}{l}\text { Japan } \\
(\mathrm{mg} / \mathrm{kg})\end{array}$ & $\begin{array}{l}\text { Korea } \\
(\mathrm{mg} / \mathrm{kg})\end{array}$ & $\begin{array}{l}\text { European Union } \\
(\mathrm{mg} / \mathrm{kg})\end{array}$ & $\begin{array}{l}\mathrm{CAC}^{*} \\
(\mathrm{mg} / \mathrm{kg})\end{array}$ & $\begin{array}{l}\text { Canada } \\
(\mathrm{mg} / \mathrm{kg})\end{array}$ & $\begin{array}{c}\text { America } \\
(\mathrm{mg} / \mathrm{kg})\end{array}$ \\
\hline Chlorpyrifos & 2 & 0.10 & 10 & 2.0 & 2.0 & 2.0 & - & - \\
\hline Triazophos & - & 0.02 & 0.05 & 0.02 & 0.02 & - & - & - \\
\hline Tolfenpyrad & 50 & - & 20 & 30 & & 30 & 30 & 30 \\
\hline Indoxacarb & 5 & - & - & - & 0.05 & 5 & - & - \\
\hline
\end{tabular}

* CAC: Codex Alimentarius Commission.

Table 6. Consumer exposure assessment of 4 pesticides in Pu'er tea.

\begin{tabular}{cccccccc}
\hline Pesticide & $\mathbf{C}(\mathbf{m g} / \mathbf{k g})$ & $\mathbf{D}^{\mathbf{a}} \mathbf{( g )}$ & $\mathbf{T} \mathbf{( \% )}$ & $\mathbf{B w}^{\mathbf{b}}$ & EDI & ADI (mg/kg bw) [13] & HQ \\
\hline Chlorpyrifos & 2.14 & 10 & $8.6[41]$ & 60 & $3.07 \times 10^{-5}$ & 0.01 & 0.00307 \\
Triazophos & 0.049 & 10 & $27.1[42]$ & 60 & $2.21 \times 10^{-6}$ & 0.001 & 0.00221 \\
Tolfenpyrad & 11.6 & 10 & $4.2[41]$ & 60 & $8.12 \times 10^{-5}$ & 0.006 & 0.0135 \\
Indoxacarb & 2.84 & 10 & $1.6[43]$ & 60 & $7.57 \times 10^{-6}$ & 0.01 & 0.000757 \\
\hline
\end{tabular}

${ }^{\mathrm{a}}$ Daily intake of Tieguanyin tea is $10 \mathrm{~g}$ and ${ }^{\mathrm{b}}$ the adult body weight is $60 \mathrm{~kg}$ [44].

Although it was acceptable to evaluate the risk of Pu'er tea using the HQ value of tolfenpyrad, its low ADI value (0.006) [13] indicates that its allowable daily intake was low. Considering this, and the determined results of 300 samples, in which the detection rate, mean residue level, and median residue level of tolfenpyrad were all at high levels, subsequent attention should be paid to its presence within our diets in the future.

\section{Conclusions}

In this study, four pesticides with high detection rates in Yunnan Pu'er tea were determined using optimized selection of MWCNTs, combined with QuEChERS-UHPLC/QTRAPMS/MS methods, and the effects of different purification methods on their matrix effects were compared. The LOQs for the four pesticides ranged from 0.10 to $0.50 \mu \mathrm{g} / \mathrm{kg}$, and the matrix effect ranged from -17.8 to 13.8 . The recoveries of the four pesticides in $\mathrm{Pu}$ 'er tea 
were in the range of $74.8-105.0 \%$, with the RSDs in the range of 3.9-6.6\%. The presence of the four pesticides in Pu'er tea, with a complex matrix, was strongly confirmed using QTRAP technology. This method was applied to samples taken from three areas in Yunnan in which $\mathrm{Pu}^{\prime} \mathrm{er}$ tea is commonly produced, in order to determine the presence of these four pesticides. The results show that the highest detection rate was that of tolfenpyrad, $35.7 \%$, while the lowest was indoxacarb, $5.2 \%$. The HQ of these four pesticides was far less than one, indicating that the risk was considered acceptable for the four pesticides in Pu'er tea. However, while it was acceptable to evaluate its risk using the HQ value of tolfenpyrad, according to the test results, the detection rate, average residual value and residual median value of tolfenpyrad were all at relatively high levels, and attention should be paid to its presence within the public's diet in the future.

Author Contributions: Conceptualization, T.L. and H.-C.L.; methodology, T.L. and X.-L.C.; software, J.G., M.-X.L. (Meng-Xia Li); validation, Y.-F.T. and L.C.; formal analysis, M.-X.L. (Mao-Xuan Li) and Y.-G.L.; investigation, M.-X.L. (Mao-Xuan Li) and Y.-G.L.; resources, J.G., M.-X.L. (Meng-Xia Li); data curation, J.G., M.-X.L. (Meng-Xia Li); writing-original draft preparation, T.L.; writing-review and editing, H.-C.L.; supervision, H.-C.L.; funding acquisition. All authors have read and agreed to the published version of the manuscript.

Funding: This study was supported by the Yunnan Key Research and Development (No.202102AE090021, 202002AE3200053, 2019ZG001).

Institutional Review Board Statement: Not applicable.

Informed Consent Statement: Not applicable.

Data Availability Statement: Data are contained within the article.

Conflicts of Interest: The authors declare no conflict of interest.

Sample Availability: Samples of the compounds are not available from the authors.

\section{Appendix A}

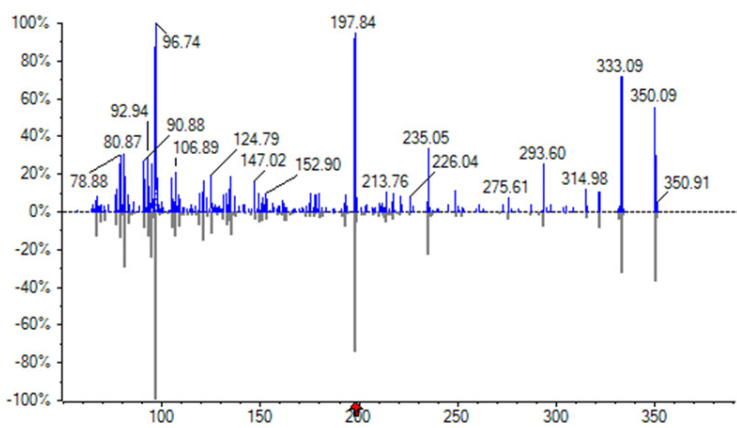

(a1)

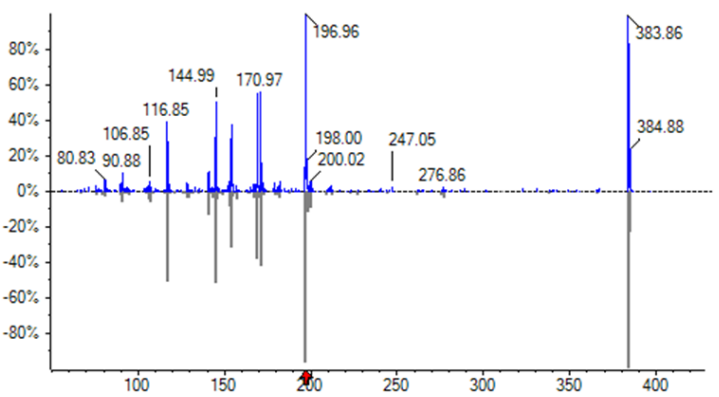

(a3)

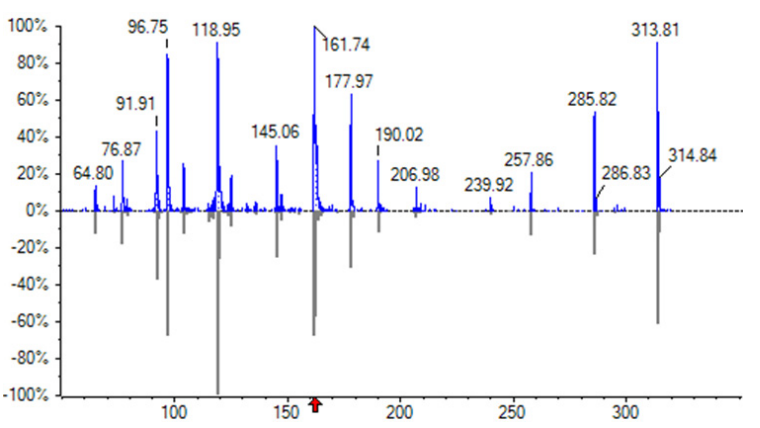

(a2)

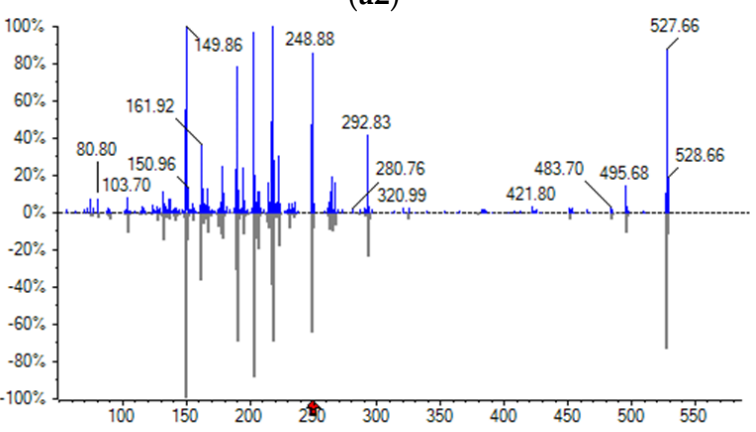

(a4)

Figure A1. The matching graph of the secondary mass spectra of the four pesticide positive samples and the standard product. (a1): chlorpyrifos; (a2): triazophos; (a3): tolfenpyrad; (a4): indoxacarb. 


\section{References}

1. Jiang, C.; Zeng, Z.; Huang, Y.; Zhang, X. Chemical compositions of Pu'er tea fermented by Eurotium Cristatum and their lipid-lowering activity. LWT-Food Sci. Technol. 2018, 98, 204-211. [CrossRef]

2. Qiong, S.; Xishuang, Y. History of Pu'er Tea and comparative study for the effect of its various extracts on lipid-lowering diet. Pak. J. Pharm. Sci. 2014, 27, 1015-1022. [PubMed]

3. Hsieh, S.-K.; Xu, J.-R.; Lin, N.-H.; Li, Y.-C.; Chen, G.-H.; Kuo, P.-C.; Chen, W.-Y.; Tzen, J.T.C. Antibacterial and laxative activities of strictinin isolated from Pu'er tea (Camellia sinensis). J. Food Drug Anal. 2016, 24, 722-729. [CrossRef] [PubMed]

4. $\quad \mathrm{Xu}, \mathrm{S}$.; Zeng, X.; Wu, H.; Shen, S.; Yang, X.; Deng, W.-W.; Ning, J. Characterizing volatile metabolites in raw Pu'er tea stored in wet-hot or dry-cold environments by performing metabolomic analysis and using the molecular sensory science approach. Food Chem. 2021, 350, 129186. [CrossRef]

5. Peng, W.; Wang, L.; Qian, Y.; Chen, T.; Dai, B.; Feng, B.; Wang, B. Discrimination of Unfermented Pu'er Tea Aroma of Different Years Based on Electronic Nose. Agric. Res. 2017, 6, 436-442. [CrossRef]

6. Dou, Z.; Ji, M.; Wang, M.; Li, H. Empirical analysis of Pu'er tea price bubble measurement based on GSADF method. Acta. Agr. Scand. B.-S. P. 2021, 71, 81-90. [CrossRef]

7. Feng, X. Building an ecological tea garden, promoting the sustainable development of Pu'er tea industry. Yunnan Agri. 2017, 10, 72-73.

8. Bai, A.; Chen, A.; Chen, W.; Liu, S.; Luo, X.; Liu, Y.; Zhang, D. Residue behavior, transfer and risk assessment of tolfenpyrad, dinotefuran and its metabolites during tea growing and tea brewing. J. Sci. Food Agri. 2021, 101, 5992-6000. [CrossRef] [PubMed]

9. Wei, Q.; Mu, X.-C.; Yu, H.-Y.; Niu, C.-D.; Wang, L.-X.; Zheng, C.; Chen, Z.; Gao, C.-F. Susceptibility of Empoasca vitis (Hemiptera: Cicadellidae) populations from the main tea-growing regions of China to thirteen insecticides. Crop. Prot. 2017, 96, 204-210. [CrossRef]

10. Liu, T.-X.; Sparks, A.N., Jr.; Chen, W.; Liang, G.-M.; Brister, C. Toxicity, persistence, and efficacy of indoxacarb on cabbage looper (Lepidoptera: Noctuidae) on cabbage. J. Econ. Entomol. 2002, 95, 360-367. [CrossRef]

11. Hikiji, W.; Yamaguchi, K.; Saka, K.; Hayashida, M.; Ohno, Y.; Fukunaga, T. Acute fatal poisoning with Tolfenpyrad. J. Forensic. Leg. Med. 2013, 20, 962-964. [CrossRef]

12. Ministry of Agriculture and Rural Affairs of the People's Republic of China. List of Prohibited and Restricted Pesticides. Available online: http:/ / www.zzys.moa.gov.cn/gzdt/201911/t20191129_6332604.htm (accessed on 7 January 2022).

13. GB 2763-2021. National Food safety Standard-In Maximum Residue Limits for Pesticides in Food; China Agriculture Press: Beijing, China, 2021; pp. 95-97, 281-282, 359-360, 365.

14. Dong, X.; Lan, T.; Tian, X.; Li, Y.; Zhao, Y.; Zong, Q.; Liu, S.; Pan, C. Simultaneous determination of 14 pesticide residues in tea by multi-plug filtration cleanup combined with LC-MS/MS. J. Environ. Sci. Heal. B. 2021, 56, 771-781. [CrossRef] [PubMed]

15. Huang, Y.; Shi, T.; Luo, X.; Xiong, H.; Min, F.; Chen, Y.; Nie, S.; Xie, M. Determination of multi-pesticide residues in green tea with a modified QuEChERS protocol coupled to HPLC-MS/MS. Food Chem. 2019, 275, 255-264. [CrossRef] [PubMed]

16. Harischandra, N.R.; Pallavi, M.S.; Bheemanna, M.; PavanKumar, K.; Chandra Sekhara Reddy, V.; Udaykumar, N.R.; Paramasivam, M.; Yadav, S. Simultaneous determination of 79 pesticides in pigeonpea grains using GC-MS/MS and LC-MS/MS. Food Chem. 2021, 347, 128986. [CrossRef]

17. Zhu, B.; Xu, X.; Luo, J.; Jin, S.; Chen, W.; Liu, Z.; Tian, C. Simultaneous determination of 131 pesticides in tea by on-line GPC-GC-MS/MS using graphitized multi-walled carbon nanotubes as dispersive solid phase extraction sorbent. Food Chem. 2019, 276, 202-208. [CrossRef] [PubMed]

18. Yao, Q.; Li, J.; Yan, S.a.; Huang, M.; Lin, Q. Occurrence of pesticides in white tea and a corresponding risk exposure assessment for the different residents in Fujian, China. J. Food Sci. 2021, 86, 3743-3754. [CrossRef] [PubMed]

19. Beneta, A.; Mutavdžić Pavlović, D.; Periša, I.; Petrović, M. Multiresidue GC-MS/MS pesticide analysis for evaluation of tea and herbal infusion safety. Int. J. Environ. An. Chem. 2018, 98, 987-1004. [CrossRef]

20. Lv, S.; Wu, X.; Guan, J.; Yan, Y.; Ge, M.; Zhu, G. Quantification and Confirmation of Zearalenone Using a LC-MS/MS QTRAP System in Multiple Reaction Monitoring and Enhanced Product Ion Scan Modes. Food Anal. Method. 2021, 14, 1-9. [CrossRef]

21. Du, Q.; Zhang, Y.; Wang, J.; Chang, J.; Wang, A.; Ren, X.; Liu, B. Quantitative analysis of 17 hypoglycemic drugs in fingerprints using ultra-high-performance liquid chromatography/tandem hybrid triple quadrupole linear ion trap mass spectrometry. Rapid Commun. Mass. Sp. 2022, 36, e9199. [CrossRef]

22. Sadowska-Rociek, A.; Surma, M.; Cieślik, E. Comparison of different modifications on QuEChERS sample preparation method for PAHs determination in black, green, red and white tea. Environ. Sci. Pollut. R. 2014, 21, 1326-1338. [CrossRef]

23. $\mathrm{Wu}, \mathrm{C}$-C. Multiresidue method for the determination of pesticides in Oolong tea using QuEChERS by gas chromatography-triple quadrupole tandem mass spectrometry. Food Chem. 2017, 229, 580-587. [CrossRef]

24. Jiao, W.; Xiao, Y.; Qian, X.; Tong, M.; Hu, Y.; Hou, R.; Hua, R. Optimized combination of dilution and refined QuEChERS to overcome matrix effects of six types of tea for determination eight neonicotinoid insecticides by ultra performance liquid chromatography-electrospray tandem mass spectrometry. Food Chem. 2016, 210, 26-34. [CrossRef]

25. Zhang, X.; Mobley, N.; Zhang, J.; Zheng, X.; Lu, L.; Ragin, O.; Smith, C. Analysis of agricultural residues on tea using d-SPE sample preparation with GC-NCI-MS and UHPLC-MS/MS. J. Agri. Food Chem. 2010, 58, 11553-11560. [CrossRef] [PubMed]

26. Li, Y.; Wang, Z.; Gao, F.; Song, D.; Lu, X. Selection of representative matrices for the multiresidue analysis of pesticides in tea by GC-MS/MS. Anal. Methods 2018, 10, 855-866. [CrossRef] 
27. Li, Y.; Pang, G.-F.; Fan, C.-L.; Chen, X. Hierarchical Cluster Analysis of Matrix Effects on 110 Pesticide Residues in 28 Tea Matrixes. J. AOAC Int. 2013, 96, 1453-1465. [CrossRef] [PubMed]

28. Yaqub, G.; Ilyas, F.; Idrees, M.; Mariyam, V. Monitoring and risk assessment due to presence of heavy metals and pesticides in tea samples. Food Sci. Techol. 2018, 38, 625-628. [CrossRef]

29. Chen, C.; Qian, Y.; Chen, Q.; Tao, C.; Li, C.; Li, Y. Evaluation of pesticide residues in fruits and vegetables from Xiamen, China. Food Control 2011, 22, 1114-1120. [CrossRef]

30. GB/T 8302-2013. Tea-Sampling; China Agriculture Press: Beijing, China, 2013.

31. European Commission Directorate-General for Health and Food Safety. European Commission Guidance Document on Analytical Quality Control and Method Validation Procedures for Pesticides Residues and Analysis in Food and Feed. Document No. SANTE/12682/2019. 2019. Available online: https://www.eurl-pesticides.eu/userfiles/file/EurlALL/AqcGuidance_SANTE_ 2019_12682.pdf (accessed on 7 January 2022).

32. Stahnke, H.; Kittlaus, S.; Kempe, G.n.; Alder, L. Reduction of matrix effects in liquid chromatography-electrospray ionizationmass spectrometry by dilution of the sample extracts: How much dilution is needed? Anal. Chem. 2012, 84, 1474-1482. [CrossRef] [PubMed]

33. New, L.-S.; Chan, E.C.Y. Evaluation of BEH C18, BEH HILIC, and HSS T3 (C18) Column Chemistries for the UPLC-MS-MS Analysis of Glutathione, Glutathione Disulfide, and Ophthalmic Acid in Mouse Liver and Human Plasma. J. Chromatogr. Sci. 2008, 46, 209-214. [CrossRef] [PubMed]

34. Xiao, M.; Chen, H.; Shi, Z.; Feng, Y.; Rui, W.J.A.M. Rapid and reliable method for analysis of raw and honey-processed astragalus by UPLC/ESI-Q-TOF-MS using HSS T3 columns. Anal. Methods 2014, 6, 8045-8054. [CrossRef]

35. Han, C.; Hu, B.; Chen, S.; Wang, N.; Hou, J.; Jin, N.; Shen, Y. Determination of Xinjunan pesticide residue in foodstuffs of plant origin by a modified QuEChERS method and ultra performance liquid chromatography-tandem mass spectrometry. LWT 2021, 151, 112101. [CrossRef]

36. Gao, G.; Chen, H.; Zhu, L.; Chai, Y.; Ma, G.; Wang, C.; Hao, Z.; Liu, X.; Lu, C. Simultaneous determination of bisphenol A and tetrabromobisphenol A in tea using a modified QuEChERS sample preparation method coupled with liquid chromatographytandem mass spectrometry. Anal. Methods 2017, 9, 6769-6776. [CrossRef]

37. Huang, Z.; Zhang, Y.; Wang, L.; Ding, L.; Wang, M.; Yan, H.; Li, Y.; Zhu, S. Simultaneous determination of 103 pesticide residues in tea samples by LC-MS/MS. J. Sep. Sci. 2009, 32, 1294-1301. [CrossRef]

38. Wang, K.; Zhao, L.; Zhang, C.; Zhang, H.; Lian, K. Determination of 12 insect growth regulator residues in foods of different matrixes by modified QuEChERS and UPLC-MS/MS. RSC Adv. 2021, 11, 12162-12171. [CrossRef]

39. Zhang, Y.; Hu, D.; Meng, X.; Shi, Q.; Li, P.; Jin, L.; Zhang, K.; Song, B. Enantioselective degradation of indoxacarb from different commercial formulations applied to tea. Chirality 2015, 27, 262-267. [CrossRef]

40. Food Codex Data. Available online: https://www.sdtdata.com/fx/fcv1/tsLibIndex (accessed on 7 January 2022).

41. Wang, X.; Zhou, L.; Zhang, X.; Luo, F.; Chen, Z. Transfer of pesticide residue during tea brewing: Understanding the effects of pesticide's physico-chemical parameters on its transfer behavior. Food Res. Int. 2019, 121, 776-784. [CrossRef]

42. Chen, H.; Pan, M.; Pan, R.; Zhang, M.; Liu, X.; Lu, C. Transfer rates of 19 typical pesticides and the relationship with their physicochemical property. J. Agri. Food Chem. 2015, 63, 723-730. [CrossRef]

43. Chen, H.; Pan, M.; Liu, X.; Lu, C. Evaluation of transfer rates of multiple pesticides from green tea into infusion using water as pressurized liquid extraction solvent and ultra-performance liquid chromatography tandem mass spectrometry. Food Chem. 2017, 216, 1-9. [CrossRef] [PubMed]

44. Yao, Q.; Yan, S.-A.; Li, J.; Huang, M.; Lin, Q. Health risk assessment of 42 pesticide residues in Tieguanyin tea from Fujian, China. Drug Chem. Toxicol. 2020, 1-8. [CrossRef] 